\title{
An Intelligent Service-Oriented Cloud Computing Architecture
}

\author{
Ding Zhou ${ }^{1, \text { a) }}$, Jian Zhang ${ }^{1, \text { b) }}$ \\ ${ }^{1}$ Faculty of Information Technology, Beijing University of Technology, Beijing 100124, China. \\ a)zdemon@sina.cn \\ b)zhangj@bjut.edu.cn
}

Keywords: Service-oriented, Cloud computing architecture

\begin{abstract}
Cloud computing is now the most popular research field. Many IT giants have built their own cloud computing infrastructure like Google App Engine, Amazon Web Service, Microsoft Azure, as well as many other open source cloud computing platforms. However, there are several issues with existing cloud computing platforms and frameworks, such as hardly migrate, a lack of standardization and customization, limited interoperability. In this paper, we put forward a cloud architecture based on SOA (Service - Oriented Architecture) to alleviate issues associated with standardization and customization. The proposed architecture not only supports traditional cloud facilities, but also provide an intelligent service layer interaction with all cloud services. With this new architecture we can easily migrate our services to different clouds and the cloud provider can afford customizable services. Furthermore, the cloud computing systems will be more flexible.
\end{abstract}

\section{INTRODUCTION}

Now everyone talking about cloud computing, what is the most dramatic change we have observed in computing since the Internet wave [1]. Cloud computing is a new compute paradigm which based on a distributed and parallel computing system that provide elastic storage resources and computing resources over the Internet. The advantages of cloud computing over traditional computing include: agility, lower entry cost, device independency, location independency, high scalability, high security, multi-tenancy, sustainability [2], internet centric, automatic adaption, payper-use and SLA (Service Level Agreement) provision [3].

An important aspect of cloud computing is cloud architecture [4] that refers to the components (e.g., service layers), subcomponents (e.g., security or message passing in service layers), and overall system organization of cloud computing [5] [6]. To effectively achieve the potential of the Cloud Computing, various Cloud Computing architectures or infrastructures have been defined. However, there are several problems with existing architectures and infrastructures, there is need for the definition of a systematic architecture of the Cloud Computing.

The paper is organized as follows. Section 2 gives some relevant concepts. Section 3 reviews related work. In section 4, we introduce our architecture and describe the layers in detail. Finally, the last section concludes the paper.

\section{RELEVANT CONCEPTS}

\section{Service-Oriented Architecture}

The SOA has been receiving considerable attention as a loosely-coupled architecture designed to meet the business needs of the organizations. According to the Organization for the Advancement of Structured Information Standards (OASIS), SOA can be defined as "a paradigm for organizing and utilizing distributed capabilities that may be under the control of different ownership domains. It provides a uniform means to offer, discover, interact with, and use capabilities to produce desired effects consistent with measurable preconditions and expectations" [7]. In the SOA model, as shown in Fig. 1, a service provider publishes his service interface via a service registry through Universal Description Discovery and Integration(UDDI) where a service request can find the service and, subsequently, may bind to the service provider. Service discovery, brokering, and reliability are important and services are usually designed to co-operate (i.e. to "composite" 
services). Services can be described and accessed using well defined service interfaces (e.g. by Web Service Description Language [8]) and via standard message-exchanging protocols (e.g. Simple Object Access Protocol [9]) and exercised by following a service contract with certain policies. This provides a loose coupling of services and ensures operational agility.

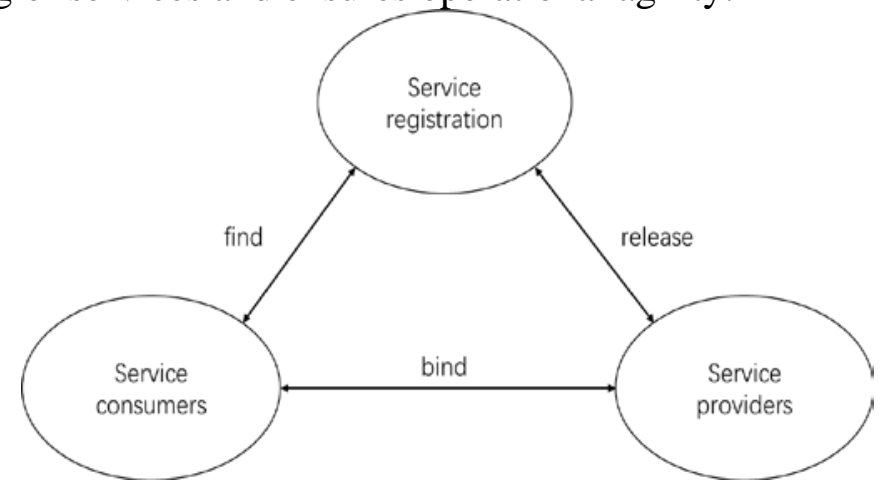

\section{FIGURE 1. SOA MODEL.}

\section{Cloud Computing}

Cloud computing is a type of Internet-based computing that provides shared computer processing resources and data to computers and other devices on demand. It is a model for enabling ubiquitous, on-demand access to a shared pool of configurable computing resources (e.g., computer networks, servers, storage, applications and services), which can be rapidly provisioned and released with minimal management effort [10][11]. In cloud computing, the following models are differentiated on the basis of horizontal scaling:

- Infrastructure-as-a-Service (IaaS) - provides a hardware platform as a service, such as Amazon EC2 or Oracle Compute Service.

- Platform-as-a-Service (PaaS) - provides a software platform as a service, such as Google Apps Engine or Oracle Java Cloud.

- Software-as-a-Service (SaaS) - allows applications and functions to be outsourced by virtualizing cloud providers' hardware and software and providing the functions as services, such as Salesforce CRM or Oracle Sales Cloud.

The key challenges of a cloud computing solution are security and quality aspects, including performance, latency, and availability. Integration, adaptation, agility, and the possible relocation of the solution play a major role during and after the implementation phase, as shown in Fig. 2.

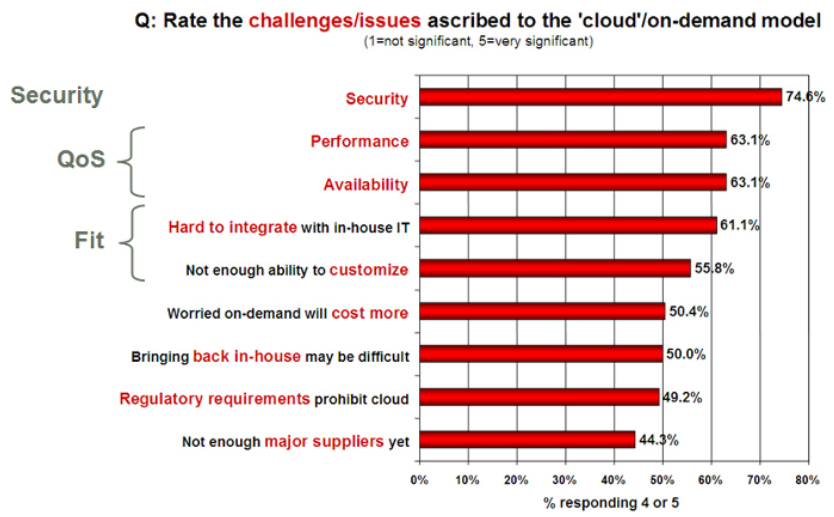

FIGURE 2. Challenges in cloud computing.

The major goal of cloud computing is to reduce cost, time-to-market and standardize platforms. Independent from the cloud deployment model and the cloud service model, a standardized and contact-based integration into the existing systems, or between cloud solutions, is essential. These aspects can be addressed with an SOA-based architecture. The integration challenge between clouds and how it can be addressed by SOA is one of the key areas. 


\section{RELATED WORKS}

Now the National Institute of Standards and Technology (NIST) is the leading organization to define cloud standards [12]. However, we do not have a generally accepted standard for cloud computing architecture. Therefore, several independent cloud computing architectures have been developed.

Some architectures proposed in [13] [14] that attempt to find a connection between SOA and cloud computing. But these cloud architectures are too theoretical and do not provide customization on each service layer.

Some other works such as [15] and [16] can be useful to implement and manage service bus which is a basis in a cloud, but none of them are prefect because they are not actually for the sake of cloud computing, and they cannot provide cloud computing basic components and layers.

Many cloud Open forums, such as OGF Open Cloud Computing Interface [17], Cloud Computing Interoperability Forum, Deltacloud [18] and Open Cloud Computing Interface [2]. The idea of most open source clouds is to provide a common interface that includes major cloud platforms.

\section{THE PROPOSED ARCHITECTURE}

\section{Cloud Computing and SOA, Hand in Hand}

Service-oriented architecture and cloud computing are reciprocal [19], SOA can be considered as a philosophy or paradigm in organizing and utilizing services and capabilities that may be under the control of different ownership domains, while cloud computing is a set of enabling technology that services a bigger, more flexible platform for enterprise to build their SOA solutions. In other words, SOA and cloud computing will coexist, complement, and support each other.

The SOA integration platform plays a key role in integrating the existing application into cloud services and between clouds. Canonical data models combined with ontology and semantics establish the basis for linking data and processes across systems and clouds in the future. But how to merge SOA and cloud computing? There are two ways to approach that is shown in Fig. 3: one is SOA completely adopt cloud computing framework to provide services, the other is SOA adopt cloud computing framework partly, however, establishing a unified standard between SOA and cloud computing service layer is what we must attention. We suppose that the best way to merge SOA and cloud computing is integrating SOA seamlessly into cloud computing platform, and then provide service with it.

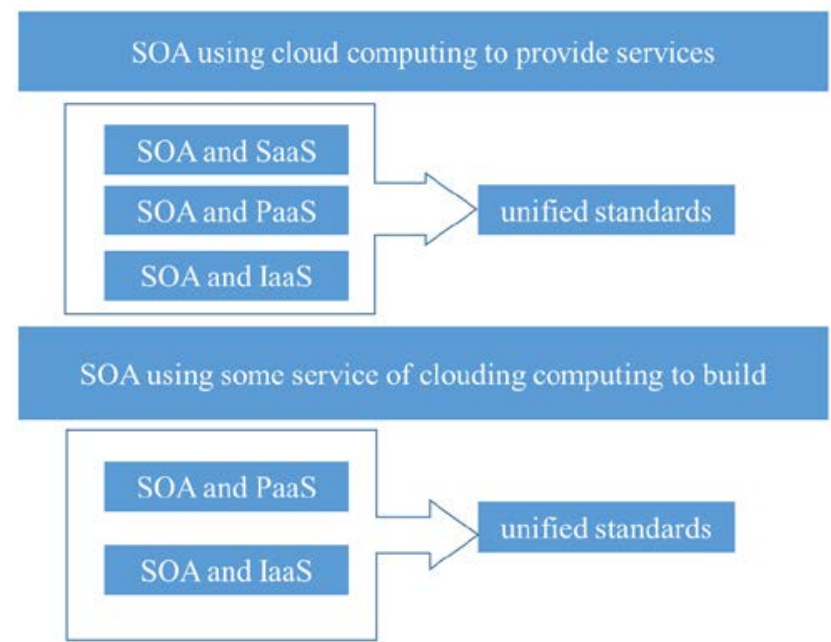

FIGURE 3. The method of merge SOA and Cloud Computing.

\section{Intelligent Service-Oriented Cloud Computing Architecture}

Our Intelligent Service-Oriented Cloud Computing Architecture is a layered architecture show in Fig 4: 


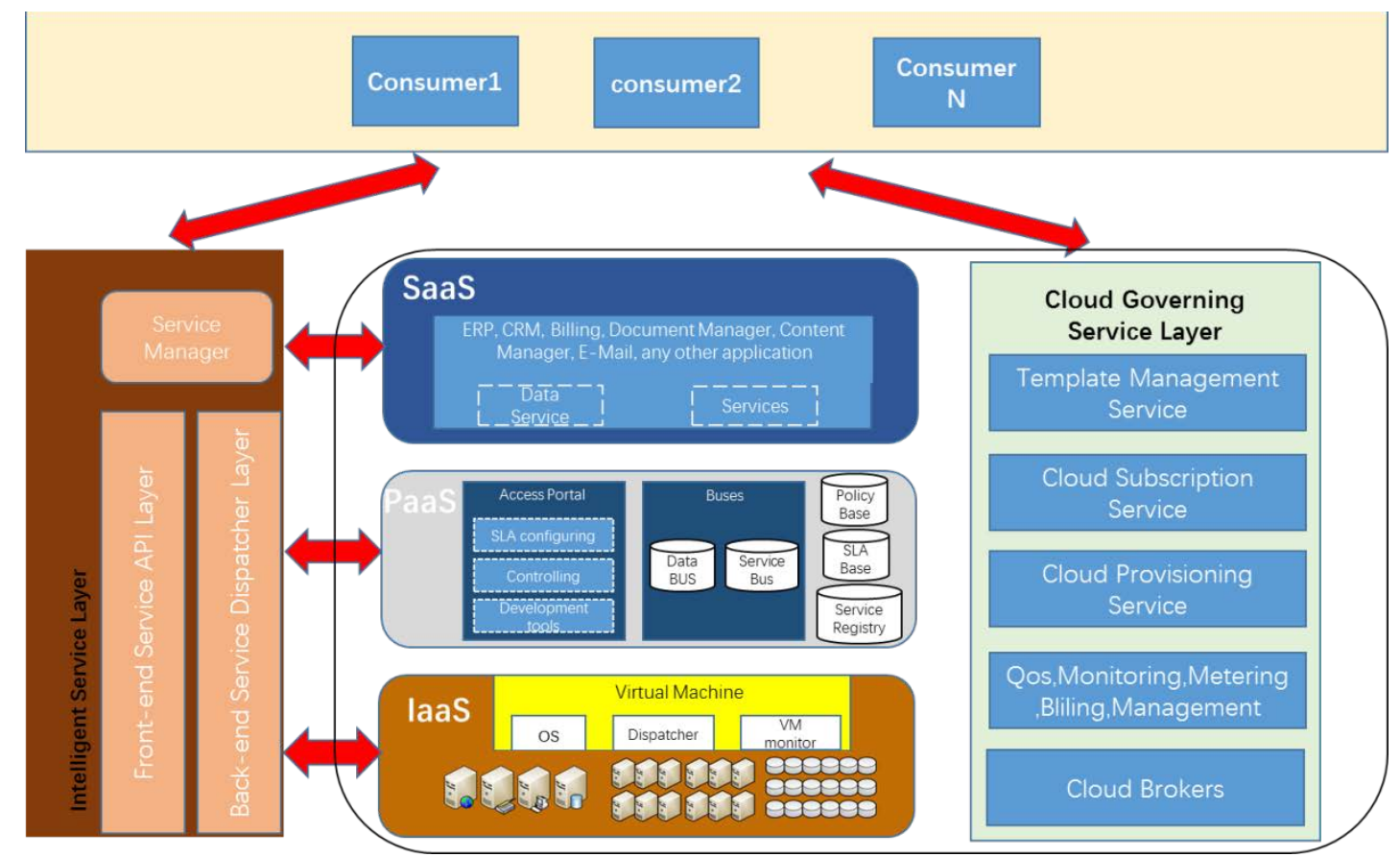

\section{1) SaaS Layer}

FIGURE 4. The proposed cloud computing architecture.

This is the layer where a consumer can access the applications, services and data services. Any application like ERP, CRM, SCM, Financial Apps, Web services, and any other software services which the user (those users which are defined in environments) are allowed to access, can be accessible through this layer. Each consumer has its own Runtime that enables the cloud to be multitenant. Each consumer can have its own application, configuration, customization and data. No other consumer can access this configuration and data without permission.

2) PaaS Layer

This layer contains Access Portal, Buses and some other Bases. Access Portal interacts with privileged users directly. One part of this portal is development tools which are in charge of defining environments and users, developing services, creating ontologies and documents, defining events and tasks. Another part is SLA configuration which allows the user to configure needed SLA for each user/group of the environment. Finally, the other part is in charge of controlling and monitoring resources. The cloud has two types of buses: service bus and data bus. Data bus is used by internal components of the cloud when they need to access some data from each base. Service bus could be used by both internal components and users. It is in relation with service registry, invokes the services and replies the results. Policy Base describes how to respond and utilize the events will be stored, every consumer can have its own policies. SLA Base is the place where the information about approved SLA for any user of any environment is registered. Search agents use this base to take the user SLA into account while searching for suitable services. Service Registry registers service descriptions and their QoS information. ESB uses this registry to search and select services.

3) IaaS Layer

This layer provides the hardware resources, virtual machines, dynamic provisioning, virtual machine manager, resource scheduler, dispatcher and any underlying requirements.

4) Intelligent Service Layer(ISL)

This layer is the heart of the framework architecture. The ISL is service-oriented, it provides a dynamic and customizable bridge between all services in cloud computing system like SaaS, PaaS, IaaS, and all cloud user groups, such as cloud providers, cloud customers, cloud developers and cloud end-users. Service providers release all their cloud services (SaaS, PaaS, IaaS) in the Service Manager of this layer. The Service Manager provide the unified interface for the consumers, so that they can find the specific services in need. Consumers could assemble these services to implement an integrated business. As shown in Fig 5, when consumers want to use a service, they will be bound to a specific service interface, then it sends a resource request to ISL. Service Manager will analyze the request and judge the corresponding availability of the resources. 


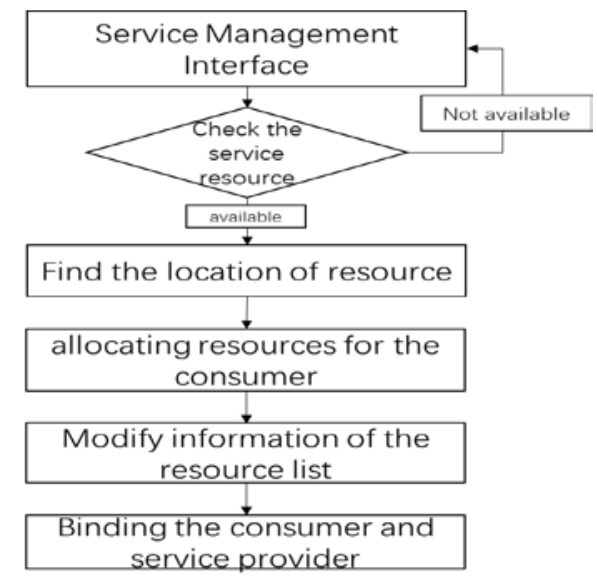

FIGURE 5. Handle process of the request.

The ISL also provides a dynamic customization on services which can be divided into sub-layers as follows: "Front-end Service API Layer (FSAL)" and "Back-end Service Dispatcher Layer (BSDL)". The FSAL provides customized cloud services to cloud consumers. The BSDL is only available to cloud providers and it interacts with all cloud services, such as all traditional services (SaaS, PaaS, IaaS). It defines a dynamic layer which can be modified and customized by a cloud provider. The BSDL can be develop alongside of existing cloud service layers. The FSAL forms the customer interface and subscriber audits.

A cloud provider defines several different services on-demand at ISL. Each defined service is a Template [20] which is integrated with one or multiple cloud service. As illustrated in Fig. 6, Cloud providers can set up, configure and provide different templates to their customers based on different service layers in a cloud computing system.

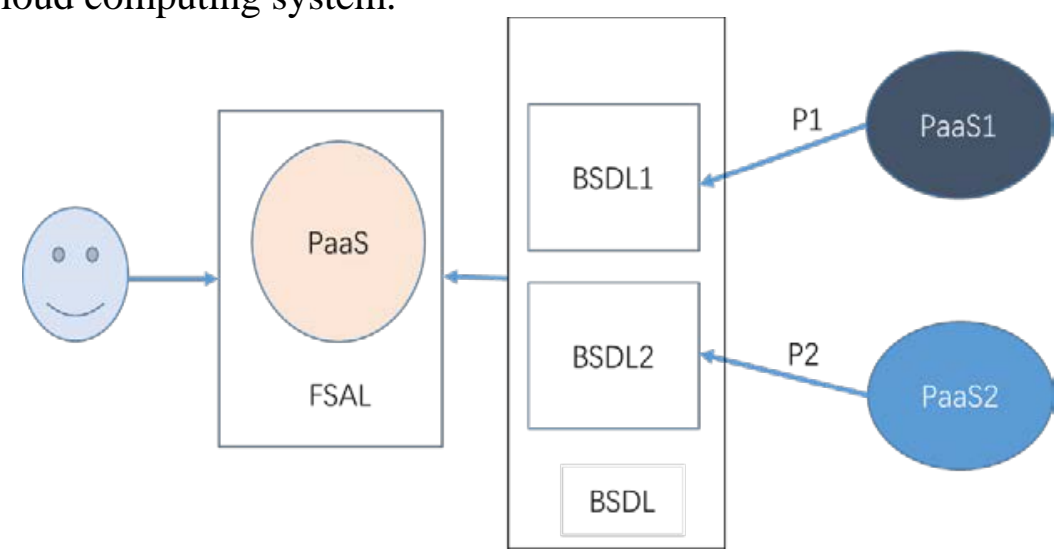

FIGURE 6. An example of a template with two different back-end for different platforms.

A cloud provider can define several templates at FSAL allows different providers to define the same template to their customers. This feature provides independent cloud services to customers who need data and applications migration from one cloud to another. Cloud providers are able to define their own cloud services with BSDL. It provides a transfer from heterogeneous services to general templates. For example, in Fig. 4, if two providers (P1 and P2) provide different PaaSs, each provider can offer a template as PaaS at FSAL. BSDL in P1 is different from BSDL in P2. Both providers should use his own BSDL to configure the back-end of his PaaS.

5) Cloud Governing Services Layer

This layer includes the following services: Template Management Service which controls the ISL to develop FSAL and BSDL. It interacts with BSDL and cloud services layer to provide a template service. Cloud Subscription Service provide a management service for defining a different type of subscriptions. Cloud Provisioning Service provides management service for resources and it provision elastic service based on Cloud Subscription Service. Quality of Service (QoS) service provides a control management on overall performance of cloud services. The cloud broker service is used to publish the cloud information, rank the services, negotiate for SLA and estimate the requests. 


\section{CONCLUSION AND FUTURE WORK}

This paper proposed a new Intelligent Service-Oriented Cloud Computing Architecture (ISOCCA) for cloud computing. The architecture which we proposed addresses the most existing cloud computing issues, such as system migration between different clouds, transfer to cloud, a lack of standardization and a lack of customization. ISOCCA provides an Intelligent Service Layer (ISL). The ISL provides simplicity these issues by defining a layer, template, with the same feature in ISL. A template is divided into front-end (FSAL) and back-end (BSDL) layers. The defined templates can be customized by a cloud provider for different groups of users. ISL also allows different cloud providers to afford the similar cloud services through a template that meets a standardization between different cloud computing systems.

In the future we will implement this architecture and deploy it on a data center. Cloud providers can set up, configure and provide different templates to their customers based on different service layers in a cloud computing system.

\section{REFERENCES}

[1].I.W. Paper, “Introduction to Cloud Computing,” 2011.

[2].Wikipedia-Cloud Computing. http://en.wikipedia.org/wiki/Cloud_computing

[3].L.M. Vaquero, L. Rodero-Merino, J. Caceres and M. Lindner, "A break in the clouds: towards a cloud definition,” SIGCOMM Comput. Commun. Rev., vol. 39, no. 1, 2008, pp. 50-55

[4].M. Bahrami and M. Singhal, The Role of Cloud Computing Architecture in Big Data, Springer International Publishing, 2015, p. 275-295.

[5].J.T. Cacioppo and G.G. Berntson, "The Affect System Architecture and Operating Characteristics," Current Directions in Psychological Science, vol. 8, no. 5, 1999, pp. 133-137.

[6].M. Bahrami, "Cloud Computing for Emerging Mobile Cloud Apps," Proc. IEEE International Conference on Mobile Cloud Computing, Services, and Engineering, 2015, pp. 4-5.

[7].C.M. Mackenzie, K. Laskey, F. Mccabe, P.F. Brown and R. Metz, "Reference model for service oriented architecture 1.0,” Oasis Standard, vol. 4, no. 6-7, 2006, pp. 8.

[8].Web Services Description Language (WSDL) for CSTA Phase III. http://www.ecmainternational.org/publications/files/ECMA-ST/Ecma-348.pdf

[9].SOAP/WSDL, WSDL, http://www.w3.org/2002/ws/

[10]. Q.F. Hassan, “Demystifying Cloud Computing,” Education”, EDUCAUSE. [Online], Retrieved October, 2011.

[11]. P. Mell and T. Grance, “The NIST definition of cloud computing," Communications of the Acm, vol. 53, no. 6, 2011, pp. 50-50.

[12]. W.T. Tsai, X. Sun and J. Balasooriya, "Service-Oriented Cloud Computing Architecture,” Proc. International Conference on Information Technology: New Generations, Itng 2010, Las Vegas, Nevada, Usa, 12-14 April, 2010, pp. 684-689.

[13]. L.J. Zhang and Q. Zhou, “CCOA: Cloud Computing Open Architecture,” Proc. IEEE International Conference on Web Services, 2009, pp. 607-616.

[14]. J.L. Marechaux, "Combining Service-Oriented Architecture and Event-Driven Architecture using an Enterprise Service Bus,” Ibm Developworks, 2006.

[15]. Z. Laliwala and S. Chaudhary, "Event-Driven Dynamic Web Services Composition: From Modeling to Implementation,” Proc. Innovations in Information Technology, 2006, pp. 1-5.

[16]. M. Behrens, M. Carlson, A. Edmonds, S. Johnston, G. Mazzafero, T. Metsch, R. Nyrén, A. Papaspyrou, A. Richardson and S. Swidler, Open Cloud Computing Interface - Infrastructure, 2011.

[17]. M. Bist, M. Wariya and A. Agarwal, "Comparing delta, open stack and Xen Cloud Platforms: A survey on open source IaaS,” Proc. IEEE International Advanced Computing Conference, 2013, pp. 96-100.

[18]. R.L. Grossman, Y. Gu, J. Mambretti, M. Sabala, A. Szalay and K. White, "An overview of the Open Science Data Cloud," Proc. ACM International Symposium on High PERFORMANCE Distributed Computing, HPDC 2010, Chicago, Illinois, Usa, June, 2010, pp. 


\section{7-384.}

[19]. D.S. Linthicum, Cloud Computing and SOA Convergence in Your Enterprise: A Step-byStep Guide, 2009, p. 470-471.

[20]. M. Bahrami, “Cloud Template, a Big Data Solution,” Computer Science(2013) 